\title{
Two-Dimensional Lithium-Ion Battery Modeling with Electrolyte and Cathode Extensions
}

\author{
Glyn F. Kennell, Richard W. Evitts \\ Department of Chemical and Biological Engineering, University of Saskatchewan, Saskatoon, Canada \\ Email: GlynKennell@usask.ca
}

Received July 3, 2012; revised August 6, 2012; accepted August 18, 2012

\begin{abstract}
A two-dimensional model for transport and the coupled electric field is applied to simulate a charging lithium-ion cell and investigate the effects of lithium concentration gradients within electrodes on cell performance. The lithium concentration gradients within electrodes are affected by the cell geometry. Two different geometries are investigated: extending the length of the electrolyte past the edges of the electrodes and extending the length of the cathode past the edge of the anode. It is found that the electrolyte extension has little impact on the behavior of the electrodes, although it does increase the effective conductivity of the electrolyte in the edge region. However, the extension of the cathode past the edge of the anode, and the possibility for electrochemical reactions on the flooded electrode edges, are both found to impact the concentration gradients of lithium in electrodes and the current distribution within the electrolyte during charging. It is found that concentration gradients of lithium within electrodes may have stronger impacts on electrolytic current distributions, depending on the level of completeness of cell charge. This is because very different gradients of electric potential are expected from similar electrode gradients of lithium concentrations at different levels of cell charge, especially for the $\mathrm{Li}_{\mathrm{x}} \mathrm{C}_{6}$ cathode investigated in this study. This leads to the prediction of significant electric potential gradients along the electrolyte length during early cell charging, and a reduced risk of lithium deposition on the cathode edge during later cell charging, as seen experimentally by others.
\end{abstract}

Keywords: Lithium-Ion Cell; Mathematical Modeling; Cathode Extension; Electrolyte Extension; Current Distributions; Electric and Concentration Fields

\section{Introduction}

Lithium-ion cells store and release energy via the emission, transport and insertion of lithium-ions from/into electrode materials at different electrochemical potentials. This difference in potential may be because the electrodes are comprised of different materials, because of an externally applied electric potential, and/or may also be because of the stoichiometric coefficient of lithium already present in the electrodes. At the ends of electrodes are edges. Lithium-ions may be produced and consumed at these edge regions if they are in contact with the electrolyte, such as when the electrolyte is flooded. At flooded electrode edges the geometry of the edges may cause multi-dimensional effects, such as concentration gradients in the electrolyte and the electrodes, and also electric potential gradients in the electrolyte. These effects are the focus of this paper.

It has previously been found that negative consequences to cell performance may arise due to the concentration gradients associated with the flooded electrode edges. These consequences include the increased risk of lithium deposition at the cathode edge region. Therefore, a cathode edge may be extended past the anode edge to reduce the likelihood of lithium deposition at the cathode edge region; however, this may result in other problems. Some of these were experimentally observed by Scott et al. $[1,2]$, and include a relatively large electric potential drop along the length of the electrolyte, parallel to the electrodes, and associated with the extended cathode edge.

West et al. [3] developed a one-dimensional model accounting for the transport in the electrolyte and electrode phases of a cell with porous insertion electrodes and a liquid electrolyte. It was demonstrated how electrolyte depletion was the principal factor limiting the discharge capacity of the system. Doyle et al. [4] presented a model for a lithium-ion cell that was implemented by considering one-dimensional transport for a galvanostatic current. It was found that the decreased lithium concentration in the composite cathode illustrated the need for higher lithium concentrations. This model was expanded by Fuller et al. [5] who considered a porous insertion anode instead of a lithium foil anode. 
Transport was considered one-dimensionally. Arora et al. [6] used the model of Fuller et al. [5] for one-dimensional lithium-ion battery predictions. They concluded that lithium deposition may occur in cells with lower excess negative electrode capacity.

Tang et al. [7] presented a two-dimensional model for the investigation of lithium deposition. They utilized a COMSOL Multiphysics ${ }^{\mathrm{TM}}$ model (based on dilute solution theory) to explain why extending the cathode edge may decrease the tendency for lithium deposition during cell charging. Some of the assumptions made by Tang et $a l$. were: constant and uniform electrolyte concentrations and conductivity, uniform anode concentration with respect to position, linearized Tafel kinetics, solid film electrodes, and electrolyte electroneutrality. Tang et al. showed that for their model a cathodic extension of 0.4 $\mathrm{mm}$ is sufficient to prevent the onset of lithium deposition. Eberman et al. [8] used a two-dimensional model based on concentrated solution theory to model the effects of a cathode under-lap (the opposite to a cathode extension). Eberman et al. used this model to conduct a sensitivity analysis of various factors on the risk for lithium deposition. They found the three most significant factors affecting the risk of deposition to be: the opencircuit potential, the size of the underlap, and the charge rate. Kennell and Evitts [9] presented a two-dimensional model for the concentrations, current distributions, and electric field as a function of time, in a lithium-ion cell. They demonstrated that it is possible to predict not only the lithium deposition at the cathode edge at later charging times, but also the large electric gradients that were experimentally observed by Scott et al. [1,2] along the electrolyte during early charging. It can be noted that all of the models described above incorporate simplified insertion kinetics when compared with models that focus on the insertion and transport of lithium inside electrode particles [10]. The research presented in this current paper uses the model of Kennell and Evitts [9] to continue the study of a lithium-ion cell and numerically predict the effects associated with equal and extended electrodes/ electrolyte.

\section{Theory and Model Implementation}

This paper is focused on results from a numerical model implemented using $\mathrm{C}++$. Lithium-ion cells were modeled using two governing equations where fluid bulk velocity has been neglected [9]:

$$
\begin{gathered}
\frac{\partial C_{i}}{\partial t}-\left(z_{i} u_{i} F \nabla \Phi\right) \cdot \nabla C_{i}=D_{i} \nabla^{2} C_{i}+S_{i} \\
\nabla^{2} \Phi=-\frac{F}{\kappa} \nabla \cdot \sum_{i} z_{i} D_{i} \nabla C_{i}-\frac{F}{\kappa} \sum_{i} z_{i} S_{i}+\frac{F}{\kappa} \frac{\partial}{\partial t}\left(\sum_{i} z_{i} C_{i}\right)
\end{gathered}
$$

Equation (1) may be used to describe the transport of species due to diffusion and electro migration. Equation (1) also contains a term for the source or sink of species due to reactions. Because Equation (1) was developed using the Control Volume technique, for use with an up-winding scheme, it omits one term that would be present in an equation developed for use with alternative methods: $-\left(z_{i} u_{i} F \nabla^{2} \Phi\right) C_{i}$. This application of Equation (1), using the Control Volume technique, also ensures the conservation of charge and mass due to transport. Equation (2) describes the Laplacian of potential due to diffusion potential, spatially separated anodic and cathodic reactions, and charge density. When this equation is applied over a time interval an assumption that the electric field will promote electroneutrality is incorporated that ensures a system of $i+1$ equations are available for solving for $i$ species concentrations and the electric field. This system of equations is advantageous when compared to equation sets containing Poisson's equation, $\nabla \cdot(\varepsilon \nabla \Phi)=-F \sum_{i} z_{i} C_{i}$, because Equation (2) is not numerically stiff. The validity and development of these equations were demonstrated by Kennell who applied these equations to several different electrochemical systems [11]. These equations were solved using $\mathrm{C}++$ and numerical techniques described elsewhere [11]. The effective diffusion coefficient was calculated [9]:

$$
D=D_{0} \varepsilon^{1.5}
$$

Values for diffusion coefficients and other model parameters are presented in Table $\mathbf{1}$.

Conductivity was assumed non-uniform with time and position inside the electrolyte:

$$
\kappa=F^{2} \sum_{i} z_{i}^{2} u_{i} C_{i}
$$

For the calculation of electro mobilities the NernstEinstein equation was used:

$$
D_{i}=R T u_{i}
$$

Predictions were conducted for a lithium-ion cell that consisted of an electrolyte sandwiched between two solid electrodes. Figure 1 shows the cell geometry and the aspects that were modified in the simulations; the extension of the cathode edge beyond the anode edge, and the electrolyte edge beyond the cathode edge, were varied. These edge extensions were conducted in the $x$-dimension, or along the length of the cell. The numerical domain was split into three parts: the cathode, the anode, and the electrolyte. These three domains were solved concurrently, where charged species were assumed to exist in the electrolyte, but not in the solid electrodes. Thus Equation (1) is written for the interior of an electrode as: 
Table 1. Cell parameters.

\begin{tabular}{lll}
\hline Electrode parameters & $\mathbf{L i}_{\mathbf{x}} \mathbf{C}_{6}$ & $\mathbf{L i}_{\mathbf{x}} \mathbf{C o} \mathbf{O}_{2}$ \\
\hline Lithium insertion rate constant $, k, \mathrm{~m}^{2.5} \cdot \mathrm{mol}^{-0.5} \cdot \mathrm{s}^{-1}$ & $4.9 \times 10^{-11}[6]$ & $2.8 \times 10^{-10}[12]$ \\
Initial stoichiometric coefficient & $0.01[7]$ & $1[7]$ \\
Maximum concentration, $C_{t}, \mathrm{~mol} \cdot \mathrm{m}^{-3}$ & $30,540[7]$ & $56,250[7]$ \\
Diffusion coefficients, $D, \mathrm{~m}^{2} \cdot \mathrm{s}^{-1}$ & $5.5 \times 10^{-14}[12]$ & $1.0 \times 10^{-11}[12]$ \\
Transfer coefficients, $\alpha_{a}, \alpha_{c}$ & $0.5[7]$ & $0.5[7]$ \\
Electrolyte parameters & & \\
Volume fraction, $\varepsilon$ & $0.55[13]$ & \\
$\mathrm{LiPF}_{6}$ initial concentration, mol $\cdot \mathrm{m}^{-3}$ & $1200[7]$ & \\
$\mathrm{Li}^{+}$diffusion coefficient in liquid phase, $D_{0}, \mathrm{~m}^{2} \cdot \mathrm{s}^{-1}$ & $8.39 \times 10^{-11}[14]$ & \\
\hline
\end{tabular}

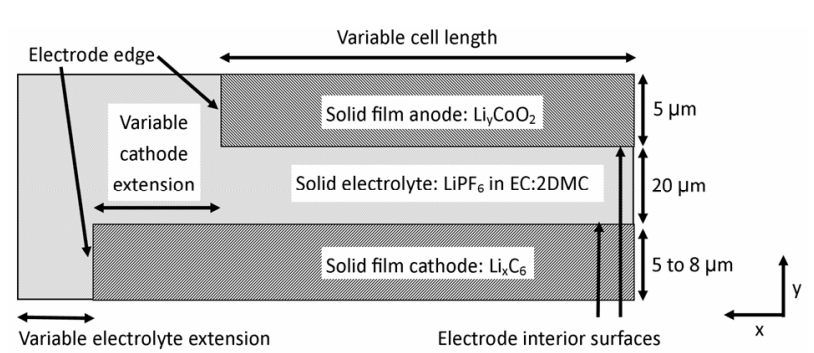

Figure 1. Cell configuration (not to scale). The $x$-dimension corresponds to the length of the cell and the $y$-dimension corresponds to the cell height.

$$
\frac{\partial C_{i}}{\partial t}=D_{i} \nabla^{2} C_{i}+S_{i}
$$

The electric potential field inside each electrode was assumed to be uniform and equal to an applied value, $\Phi_{a}$ or $\Phi_{c}$. Conductivity was also assumed constant inside each electrode. The boundary condition for all boundaries of each of the three numerical domains for Equation (1) or (6) was:

$$
\frac{\partial C_{i}}{\partial x}=0
$$

and the boundary condition used for Equation (2) at all electrolyte boundaries was:

$$
\frac{\partial \Phi}{\partial x}=0
$$

During charge and discharge, charge and mass were calculated to move between the three numerical domains via the source terms in Equations (1), (2) and (6). The following development will describe how the source terms were calculated as being dependent on electrochemical reactions, how charge and mass were conserved, and how the overall cell potential was determined.

The rates of electrochemical reactions were assumed to follow Tafel kinetics for the anode and cathode:

$$
i_{a}=i_{o, a} \exp \left(\frac{\alpha_{a, k} F}{R T}\left(\Phi_{a}-\Phi_{e}-U_{a}\right)\right)
$$

$$
i_{c}=-i_{o, c} \exp \left(-\frac{\alpha_{c, k} F}{R T}\left(\Phi_{c}-\Phi_{e}-U_{c}\right)\right)
$$

where $\Phi_{e}$ represents the electric potential of the electrolyte adjacent to the electrode. $\Phi_{e}$ was calculated from Equation (2), and was not assumed uniform with time and position. $U_{a}$ and $U_{c}$ represent the equilibrium potential of the anode and cathode respectively, as a function of lithium stoichiometric coefficient. The equilibrium potential in the anode was calculated by [12]:

$$
\begin{aligned}
& U_{a}=3.8552+1.2473\left[1-\left(\frac{C_{s}}{C_{t, a}}\right)\right]-11.152\left[1-\left(\frac{C_{s}}{C_{t, a}}\right)\right]^{2} \\
& +42.8185\left[1-\left(\frac{C_{s}}{C_{t, a}}\right)\right]^{3}-67.711\left[1-\left(\frac{C_{s}}{C_{t}}\right)\right]^{4} \\
& +42.508\left[1-\left(\frac{C_{s}}{C_{t, a}}\right)\right]^{5}-6.132 \times 10^{-4} \exp \left[7.657\left(\frac{C_{s}}{C_{t, a}}\right)^{115}\right]
\end{aligned}
$$

The equilibrium potential in the cathode was calculated by $[6]$ :

$$
\begin{aligned}
U_{c} & =0.7222+0.13868\left(\frac{C_{s}}{C_{t}}\right)+0.028952\left(\frac{C_{s}}{C_{t}}\right)^{0.5} \\
& -0.017189\left(\frac{C_{s}}{C_{t}}\right)^{-1}+0.0019144\left(\frac{C_{s}}{C_{t}}\right)^{-1.5} \\
& +0.28082 \exp \left\{15\left[0.06-\left(\frac{C_{s}}{C_{t}}\right)\right]\right\} \\
& -0.79844 \exp \left\{0.44649\left[\left(\frac{C_{s}}{C_{t}}\right)-0.92\right]\right\}
\end{aligned}
$$

The exchange current density from Equations (9) and (10) was calculated by [7]:

$$
i_{o}=F k\left(C_{t}-C_{s}\right)^{\alpha_{a}}\left(C_{s}\right)^{\alpha_{c}}(C)^{\alpha_{a}}
$$

Figure 2 shows the equilibrium potentials of the elec- 
trodes described by Equations (11) and (12) for an anode fabricated from $\mathrm{Li}_{y} \mathrm{CoO}_{2}$ and for a cathode from $\mathrm{Li}_{x} \mathrm{C}_{6}$. The solid lines in Figure 2 represent the portion of the equilibrium potentials that correspond to the stoichiometric coefficient that is likely to exist in a lithium-ion cell. In other words, the solid part of the line for $\mathrm{Li}_{\mathrm{y}} \mathrm{CoO}_{2}$ corresponds to the stoichiometric coefficient of between 0.99 and 0.58 (in the anode) and the solid part of the line for $\mathrm{Li}_{\mathrm{x}} \mathrm{C}_{6}$ corresponds to the stoichiometric coefficient of between 0.01 and 1 (in the cathode).

The electrochemical reactions were treated as source terms. If it is assumed that at the surface of the electrode the currents described by Equations (9) and (10) are perpendicular to the electrode surface, the current vector describing the electrochemical reaction rate, $\boldsymbol{i}_{i}$, is produced. This current vector may be converted into a source term for use with Equations (1), (2), and (6):

$$
S_{i}=\frac{\nabla \cdot \boldsymbol{i}_{i}}{z_{i} F}
$$

Therefore, the species produced by electrochemical reactions were introduced into the numerical procedure via the source terms in Equations (1), (2) and (6). The conservation of charge and mass across numerical boundaries was guaranteed by ensuring the sum of each electrochemical reaction along the length of the electroactive surface was equal to a prescribed current:

$$
\oint_{\text {electrode }} i_{L i} \mathrm{~d} s=I_{\text {set }} l_{\text {electrode }}
$$

Equation (15) was satisfied by modifying the rates of electrochemical reactions by varying the applied electric potentials, $\Phi_{a}$ and $\Phi_{c}$. Then, the overall cell potential was calculated by:

$$
V_{\text {cell }}=\Phi_{a}-\Phi_{c}
$$

\section{Results and Discussion}

Lithium-ion cells depend upon the transport of $\mathrm{Li}^{+}$. It may be transported through an electrolyte because of different potentials of electrodes. Different potentials may be caused by different equilibrium potentials and different potentials applied to each electrode. Equilibrium potentials depend on electrode materials and on the stoichiometric coefficient of inserted lithium. The two electrode materials investigated in this paper are $\mathrm{Li}_{y} \mathrm{CoO}_{2}$ and $\mathrm{Li}_{x} \mathrm{C}_{6}$. The equilibrium potentials corresponding to different stoichiometric coefficients in each of these materials are presented in Figure 2. Simulations presented in this paper investigate both the electric potentials established between the anode and cathode of a lithium-ion cell (along the $y$-dimension), and also the electric potentials that may exist parallel to the electrodes along the cell length ( $x$-dimension). The cell geometries investi- gated in the simulations presented in this paper are shown in Figure 1. The electrodes are considered to be solid film electrodes and the separator consists of an electrolyte with the following composition: $1.2 \mathrm{M} \mathrm{LiPF}_{6}$ in a 1:2 $\mathrm{v} / \mathrm{v}$ mixture of ethylene carbonate and dimethyl carbonate (EC:2DMC). Further details are given in Figure 1, including the locations of electrode edges and interior surfaces.

\subsection{Electrolyte Extension}

In this section predictions for lithium-ion cells with a flooded electrolyte extended past the edges of the electrodes are presented. In this section, the anode and cathode edges are located at the same cell length ( $x$-dimension). This geometry exposes the edges of both the anode and cathode to the electrolyte. Section 3.1.1 explores simulations where no electrochemical reactions occur on these electrode edges and Section 3.1.2 explores the case where electrochemical reactions occur on the electrode edges in contact with the flooded electrolyte.

\subsubsection{Equal Length Electrodes without Edge Reactions}

Figure 3 shows the predicted electric field for the case where the anode and cathode were of equal length ( $x$ dimension) and the electrolyte length was extended past the edges of the electrodes by $25 \mu \mathrm{m}$ and the cell underwent $4.37 \mathrm{Am}^{-2}$ charging for 60 seconds. The length of the electrodes incorporated into the simulation was 70 $\mu \mathrm{m}$. It was found that this length encompassed completely the multi-dimensional edge effects, for this case. It was assumed that only the interior surfaces of the electrodes were electro active. In other words, the edges of the electrodes did not emit or insert any lithium-ions. This means that the increased surface area due to electrode edges did not have an impact on the overall rate of electrochemical reactions around the edge. Therefore, in

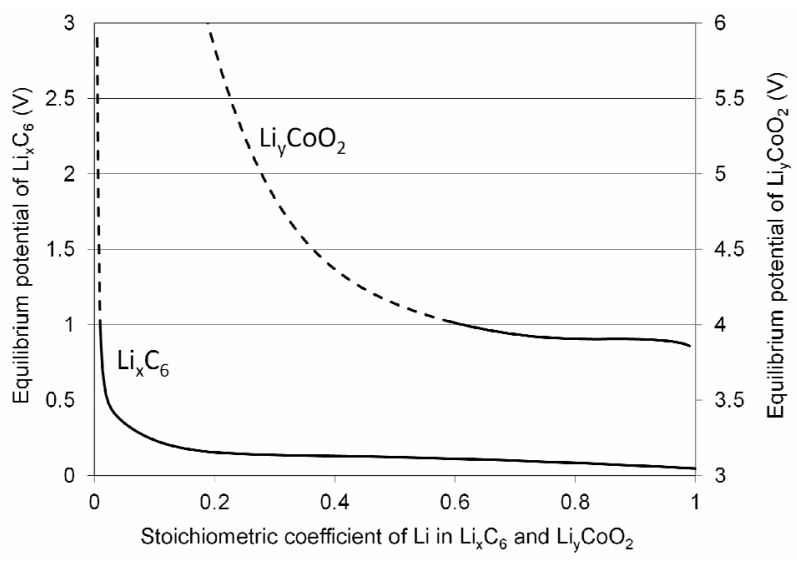

Figure 2. Equilibrium potential of electrodes as a function of stoichiometric coefficient, $x$ or $y$. Solid lines indicate stoichiometric coefficient ranges assumed in this paper. 


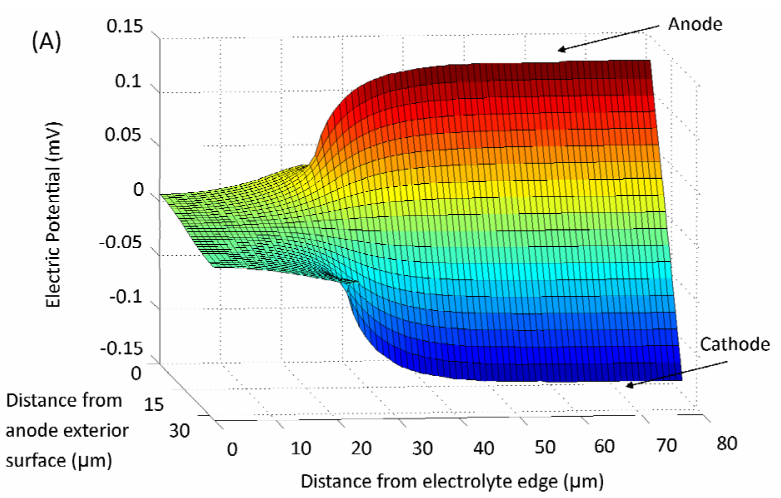

(B)

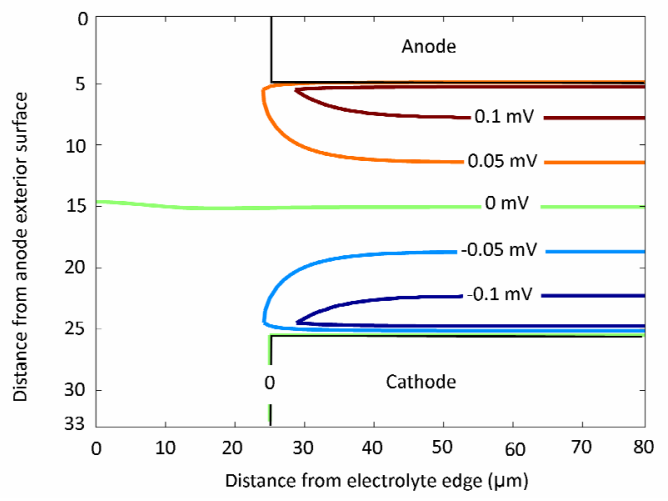

Figure 3. Predicted electric potential field for the case of equal length flooded electrodes without edge reactions after 60 seconds as (A) a surface plot and (B) a contour plot with cell geometry overlay. The height of the cathode was $8 \mu \mathrm{m}$. Simulated electrode length to bulk conditions was $70 \mu \mathrm{m}$.

this case, the main edge effect was to increase the effective conductivity of the electrolyte towards the edge, caused by the electrolyte length ( $x$-dimension) extension. The effect of this electrolyte extension on the predicted electric current distribution is shown in Figure 4. This figure demonstrates how the electric current tended to move in the $y$-dimension across the electrolyte directly from the anode to the cathode in the bulk interior of the cell (at larger distances from the edge); however, in the electrolyte nearer the edges of the electrodes it is shown that the electric current did not take the shortest path from the anode to cathode. Instead, the current tended to spread out along the cell length ( $x$-dimension) into the extended electrolyte region that would otherwise have contained no current density. In other words, the extended electrolyte region had the effect of increasing the effective conductivity of this area. This increase in effective conductivity and reduced current densities near the edge region lead to a lower electric potential gradient, as seen in Figure 3.

The rates of electrochemical reactions (Equations (9) and (10)) occurring on the electrode interior surfaces were dependent upon the electric potential adjacent to the electrode, $\Phi_{e}$. As described above, Figure 3 shows how the electric potential adjacent to the electrodes was reduced in the proximity of the edge region, including on the interior electrode surfaces, due to the increased effective conductivity of the extended electrolyte region. In other words, an increased effective conductivity due to an extended electrolyte may decrease the potential gradient across the electrolyte (in the $y$-dimension) and this may cause an increase in electrochemical reaction rates on the interior surface near the electrode edges. This effect was seen in the simulations. However, this effect was extremely small, as can be seen in Figure 5, which shows the concentration of lithium inserted into the cathode after a full hour of $1 \mathrm{C}$ charging; the increase in lithium concentration near the cathode edge/tip was so small that it is unobservable in this figure. Hence, it can be concluded that the electrolyte extension does not have a perceivable effect on electrode concentrations at the conditions examined, but it does increase the effective conductivity near the edge region.

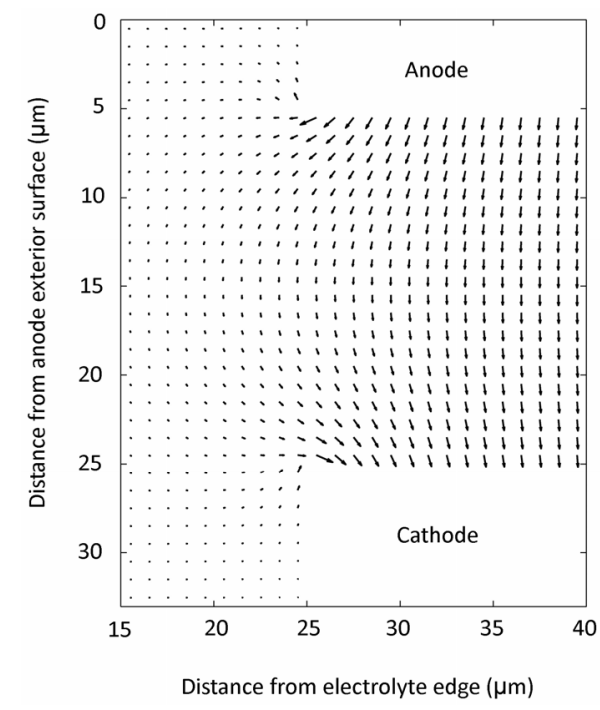

Figure 4. Predicted electric current distribution near electrode edges for the case of equal length flooded electrodes without edge reactions after 60 seconds.

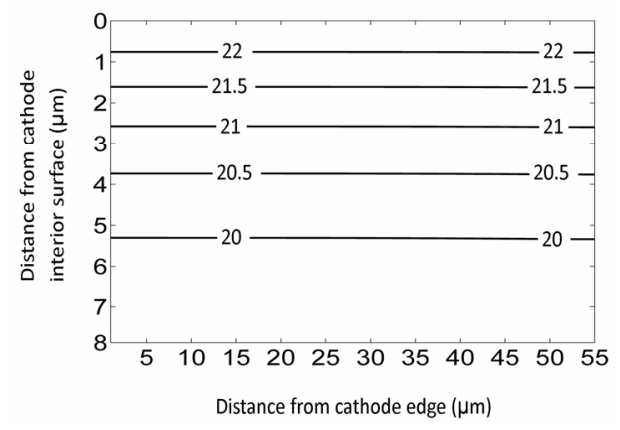

Figure 5. Predicted cathode concentration for the case of cathode height of $8 \mu \mathrm{m}$ and equal length flooded electrodes after 1 hour of charging and no edge reactions. 


\subsubsection{Equal Length Electrodes with Edge Reactions}

Figure 6 shows the predicted electric field for the case of two equal height ( $5 \mu \mathrm{m}$ in $y$-dimension) and equal length electrodes with an electrolyte length extension of $25 \mu \mathrm{m}$ after 60 seconds of $4.37 \mathrm{Am}^{-2}$ charging. For this simulation, it was assumed that the electrochemical reactions occurred along the complete surfaces of the electrodes in contact with the electrolyte, including the electrode interior surfaces and electrode edges. It is important to note that the scale changes along the abscissa in Figure 6. From this figure it may be seen that the effect of the edge reactions (when the height of both electrodes was $5 \mu \mathrm{m}$ ) was to raise the electric potential near the edges above that in the bulk region. The reason for this increase was associated with gradients of lithium stoichiometric coefficient in the electrodes towards the edges. This phenomenon is explained in the next paragraph.

The electric potential was elevated along the electrolyte length towards the electrode edges because the overall rate of anodic half reactions in this region was predicted to be greater than the overall rate of cathodic reactions. In other words, in this edge region, the anode was producing more electric current than the cathode was

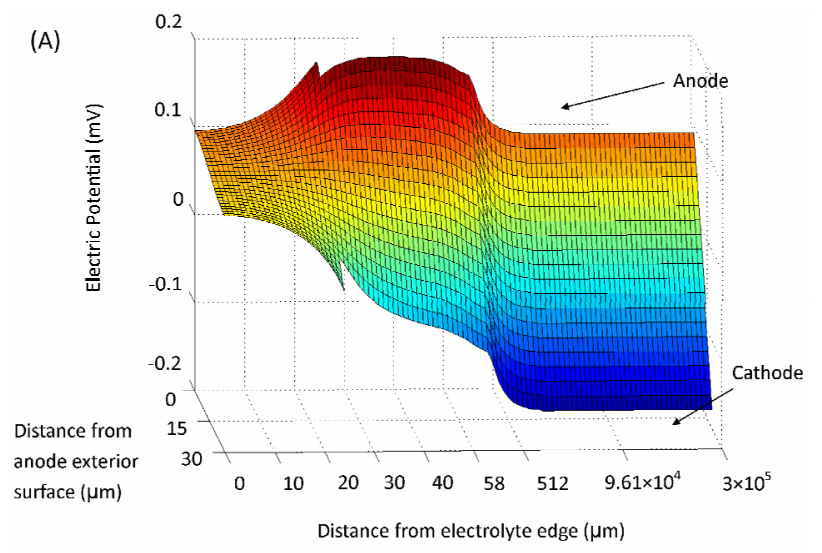

(B)

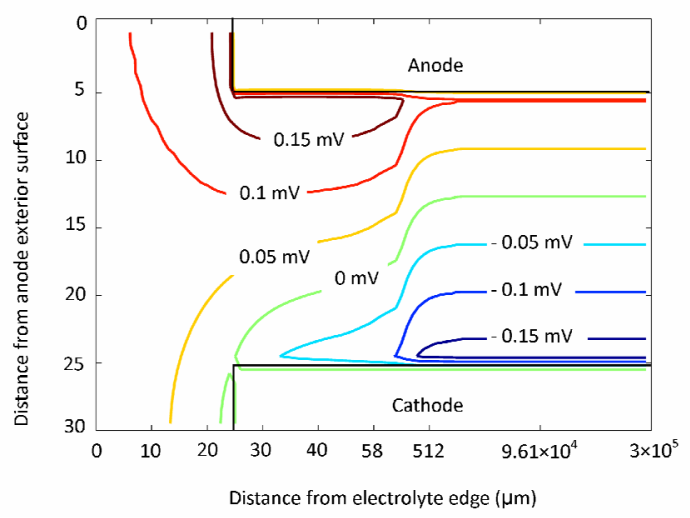

Figure 6. Predicted electric potential field for the case of equal length flooded electrodes with edge reactions after 60 seconds of charging as (A) a surface plot and (B) a contour plot with cell geometry overlay. consuming. This excess electric current then had to migrate along the length of the electrolyte, parallel to the electrodes, towards the bulk cell. This predicted electric current distribution is shown in Figure 7. This figure shows electric current emanating from the anode interior surface and the anode edge. A significant portion of this electric current flowed into the extended electrolyte region, taking advantage of the increased effective conductivity in this area. This electric current flowed towards the cathode interior surface and edge, where lithium was inserted into the cathode. The relative magnitudes of the currents associated with the tips of the anode and cathode edges can also be observed in Figure 7.

In Figure 7, the arrow depicting the current flowing/inserting into the cathode tip is approximately half of the size of the arrow depicting the current emanating from the anode. The difference in magnitude of these two currents is due to the dependence of the rates of electrochemical reactions on the equilibrium potentials, $U_{a}$ and $U_{c}$. When the charging of the cell is started, the stoichiometric coefficient of lithium in the anode is very close to unity and is represented by the right-hand portion of the solid line in Figure 2. This represents only a slight gradient of potential for a gradient in stoichiometric coefficient. In other words, this equilibrium potential does not change significantly for a concentration gradient inside the electrode, and electrochemical reaction rates will not change drastically for a gradient in potential. Therefore, for this case a higher current will emanate from the anode tip that is more dependent on the tip surface area than it is by the concentration gradient within the electrode. The opposite is true of the cathode tip. At the start of cell charging, the stoichiometric coefficient

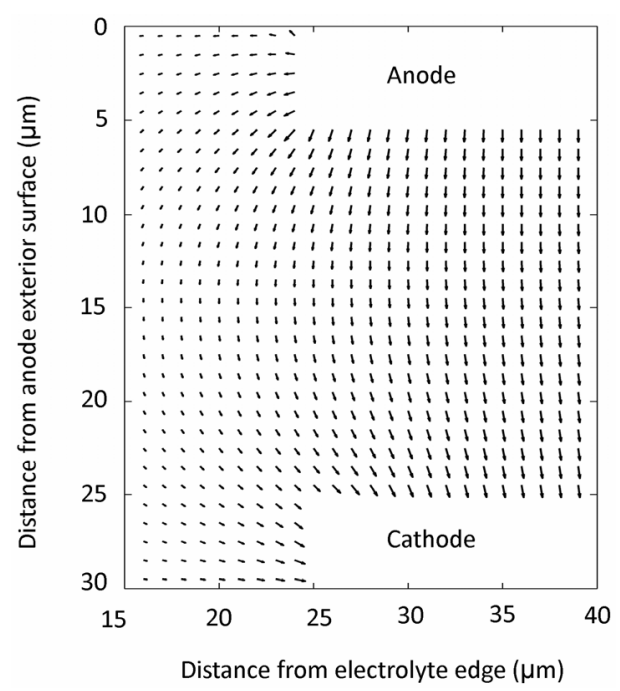

Figure 7. Predicted electric current distribution near electrode edges for the case of equal length flooded electrodes with edge reactions after 60 seconds. The height of both electrodes is $\mathbf{5} \boldsymbol{\mu m}$. 
inside the cathode is small and is shown by the left side of the $\mathrm{Li}_{x} \mathrm{C}_{6}$ solid line in Figure 2. As is evident, there is a very large gradient of potential associated with a small change in stoichiometric coefficient in this region. Therefore, the rates of electrochemical reactions would be greatly affected by a gradient of lithium concentration in the electrode. Although the larger surface area at the cathode tip may promote an increased rate of lithium insertion into the tip area, the large change in equilibrium potential that this would cause prevents this increase. Instead, the extra current produced at the anode tip and edge must flow along a different path, along the electrolyte length (in the $x$-dimension) adjacent to the cathode, in a manner that balances transport and concentration gradients in the electrolyte with concentration and equilibrium potentials in the electrodes. The resulting concentration gradients within the electrodes are discussed below.

Figure 8 shows the concentration gradients in the region of the cathode edge after 60 seconds of cell charging. The lengthwise gradients were restricted to within 5 $\mu \mathrm{m}$ of the edge. This is a smaller region than for the gradients in the anode, shown in Figure 9, where the lengthwise gradients extended $25 \mu \mathrm{m}$ from the anode edge. This reinforces the concept that stoichiometric gradients occur in the anode during early cell charging, but not in the cathode because of the large equilibrium potential gradients that this would cause. In other words, the electrochemical reaction rate of lithium production at the anode is not greatly affected by the anodic lithium stoichiometric coefficient; the opposite is true of the cathode (during early charging). Because the numerical model used a non-uniform mesh, the length of the bulk cell that was modeled was long enough so that the microscopic phenomena occurring at the electrode edges would not have a significant effect on the macroscopic bulk cell and overall cell potential. Because the model balances the two-dimensional potential field at the edges with the bulk cell potential, the model predicts where the excess current from the anode edge is inserted into the cathode. Figure 10 shows the current density that occurred on the surfaces of both the anode and cathode as a

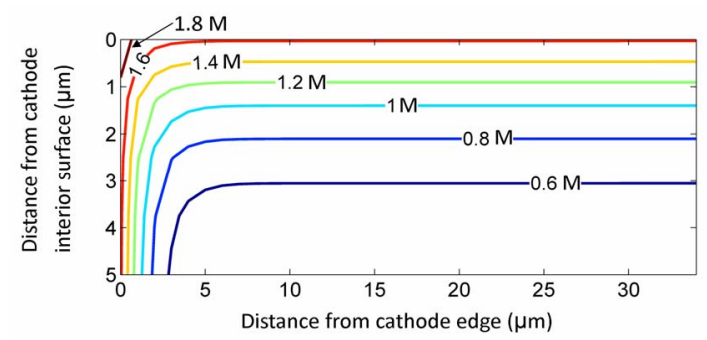

Figure 8. Predicted cathode concentration for the case of equal length flooded electrodes with edge reactions after 60 seconds of $4.37 \mathrm{Am}^{-2}$ charging.

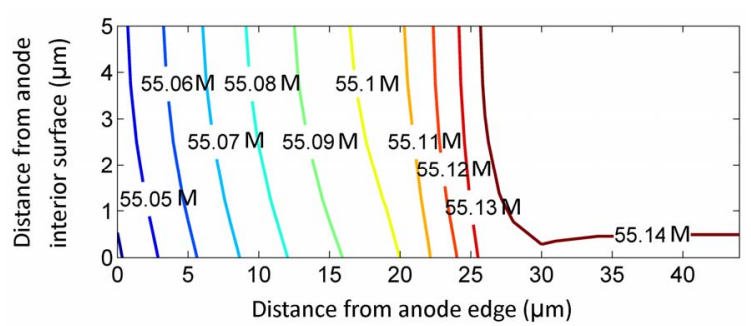

Figure 9. Predicted anode concentration for the case of equal length flooded electrodes with edge reactions after 60 seconds of $4.37 \mathrm{Am}^{-2}$ charging.

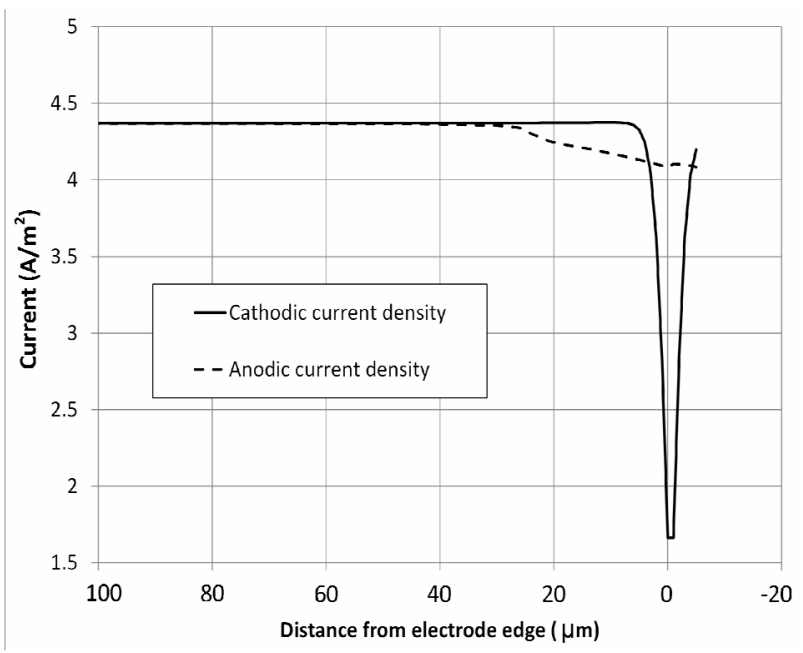

Figure 10. Predicted electrochemical reaction rate of lithium dissolution or insertion along surface for the case of equal length flooded electrodes with edge reactions after 60 seconds of $4.37 \mathrm{Am}^{-2}$ charging.

function of distance from the electrode edge (positive distances correspond to locations on the electrode interior surface, away from the edge, and negative distances correspond to distances away from the electrode tip, along the electrode edge itself). Figure 10 shows a reduction in the cathodic current density at the electrode tip, of approximately $65 \%$ of the bulk value, and this is necessary in order to avoid large stoichiometric lithium gradients in the cathode (as described above). The anodic reaction rate suffers only a small reduction at the tip, as shown in Figure 10. However, since the overall production and consumption of $\mathrm{Li}^{+}$must be equal for the entire cell, this excess $\mathrm{Li}^{+}$produced at the electrode edges must be consumed elsewhere. Figure 10 shows at distances of approximately $0.01 \mathrm{~mm}$ to $0.02 \mathrm{~mm}$ from the edge the anodic reaction rate was predicted to be less than the cathodic reaction rate. Therefore, it is in this region that the excess current from the edge region was inserted into the cathode. This avoided steep lithium concentration gradients towards the cathode edge, and instead balanced these gradients with iR drops and concentration effects in the electrolyte. 
Figure 11 shows the predictions for a cell with similar geometry to the one used in the previous predictions, but with a cathode height of $8 \mu \mathrm{m}$. This increase in cathode height decreases the concentration gradients within the cathode caused by lithium diffusion from the surfaces. The prediction showed that an increase in cathode height decreases the values of electric potential towards the electrode edges. Figure 12 shows the electric current distribution for this case. Figure 12 displays the current that was drawn parallel to the electrodes (along its length) into the edge area to compensate for the additional lithium inserted into the cathode in this area. Hence, during early cell charging, the rate of lithium insertion into the cathode is primarily determined by the concentration gradients within the cathode, and the rate of lithium emission from the anode is determined by anode surface area and electrolyte potential gradients.

\subsection{Extended Cathodes}

Large potential drops due to concentration gradients within the cathode have been seen experimentally when
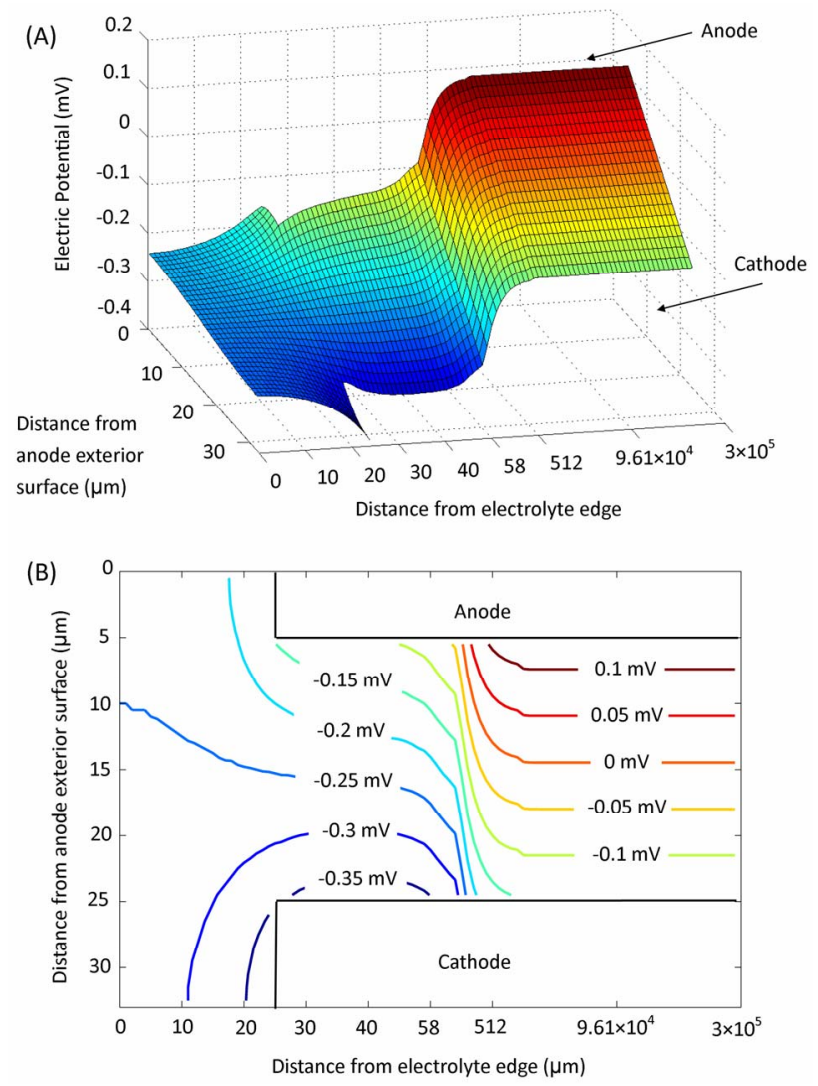

Figure 11. Predicted electric potential field for the case of equal length flooded electrodes with edge reactions after 60 seconds of charging as (A) a surface plot and (B) a contour plot with cell geometry overlay. The height of the cathode was $8 \mu \mathrm{m}$. The electrolyte length was extended past the edge of the electrodes by $25 \mu \mathrm{m}$.

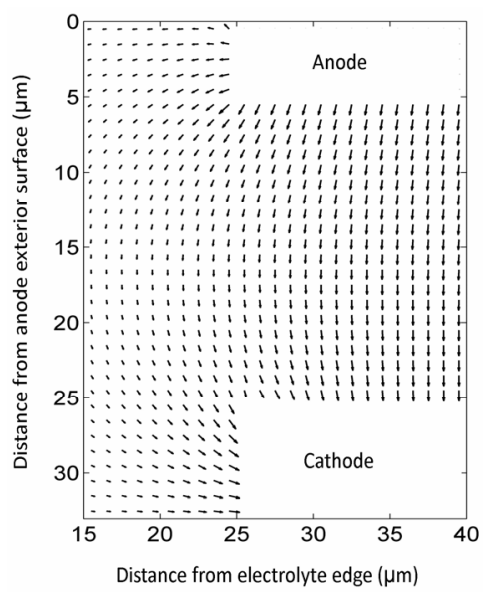

Figure 12. Predicted electric current distribution near electrode edges for the case of equal length flooded electrodes and $8 \mu \mathrm{m}$ height cathode after 60 seconds of charging.

the cathode edge is extended (in the $x$-dimension) significantly past the anode edge (Scott et al. [1,2]). The cathode edge may be extended in order to prevent higher levels of lithium concentration at the tip/edge that may be detrimental to the cell. The model presented in this paper does predict these damaging levels of lithium concentration, and the resulting lithium deposition, at the cathode tip/edge; however, these high concentrations of lithium are more likely to occur in the cathode towards the end of cell charging when the stoichiometric coefficient of lithium in $\mathrm{Li}_{\mathrm{x}} \mathrm{C}_{6}$ is almost unity, rather than at the beginning of cell charging. The relationship between the equilibrium potential and stoichiometric coefficient of lithium in $\mathrm{Li}_{\mathrm{x}} \mathrm{C}_{6}$ of an almost completely charged cell is shown in Figure 2 (for stoichiometric coefficients approaching unity). The potential gradient caused by the $\mathrm{Li}_{\mathrm{x}} \mathrm{C}_{6}$ stoichiometric coefficient gradient is much less for coefficients approaching unity (a fully charged cell) than for coefficients approaching zero (an uncharged cell). These different equilibrium potential gradients for an uncharged and charged cathode result in the possibility for larger lithium concentration gradients in a cathode approaching a full charge. In other words, if the difference in stoichiometric coefficient of lithium in an electrode causes a large equilibrium potential gradient, a large electric potential gradient may be apparent in the electrolyte, as seen by Scott et al. [1,2]. If the cell is in a state of charge whereby a large difference in stoichiometric coefficient (with length) does not cause a large equilibrium potential gradient, then large electric potential gradients may not be seen in the electrolyte; however, large concentration gradients in the electrode may then be possible, along with electrode over-saturation and lithium deposition at regions of high surface area, as seen in the numerical simulations of Tang et al. [7]. The model presented in this paper predicts both such phenomena. For 
example, Figure 13 shows the concentration profile for the cathode of $6 \mu \mathrm{m}$ height and equal electrode length cell having undergone $4.37 \mathrm{Am}^{-2}$ charging for one hour. Figure 13 shows that although much of the cathode concentration was significantly below the maximum concentration of $30.5 \mathrm{M}$, the tip region was above this concentration and lithium deposition here is likely. Figure $\mathbf{1 4}$ shows the predicted electric field for the case where the length of the cathode was extended past the edge of the anode by $1.75 \mathrm{~cm}$, after 100 seconds of charging at 2 $\mathrm{Am}^{-2}$, corresponding to the current density utilized by Scott et al. [1]. The height of the cathode was $8 \mu \mathrm{m}$ and the initial stoichiometric coefficient in the cathode was 0.0025 . Figure 14 shows a predicted electric field that has a minimum of approximately $-0.4 \mathrm{~V}$ with respect to the bulk values. This is about half of the maximum experimental value seen by Scott et al. [1] after 90 seconds for a lithium ion cell with a cathode extension undergoing charging of a similar current density. However, many cell parameters were unpublished by Scott et al. and the predictions given here are for solid electrodes, not porous ones. Of interest in this case is not a direct comparison with experimental data, but instead, the trends caused by lithium gradients in the electrodes and resulting effects are examined below. For the simulation presented in Figure 14 the concentration of lithium in both electrodes changed with position and time. Because the concentration of lithium is non-uniform with position, potential gradients were evident in the electrolyte. The effect of the lithium electrode gradients on the cell are investigated through an examination of the rates of electrochemical reactions predicted to occur on electrode surfaces. Figure 15 shows the rate of anodic currents that emanated from the anode from the simulation presented in Figure 14 as a function of position at different times. Positive distance values represent the interior surface of the anode and negative values the small edge region. Because at larger distances along the cell length, away from the anode edge, the current was predicted to remain constant, this data was not presented as part of this figure. Figure 15 shows that at early charging times (100 s and $500 \mathrm{~s}$ ) the highest rates of current were drawn from regions close to the anode edge. The anode emanated cur-

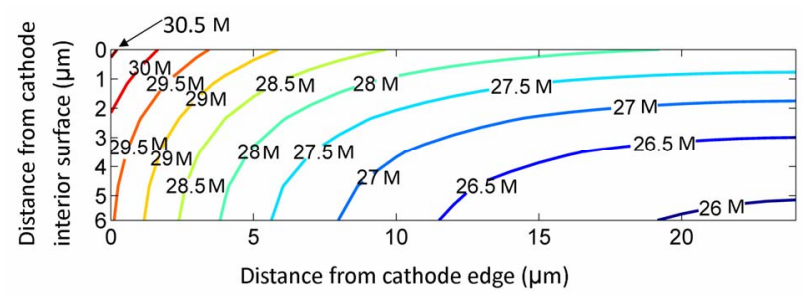

Figure 13. Predicted cathode concentration for the case of equal length flooded electrodes with edge reactions after 1 hour of $4.37 \mathrm{Am}^{-2}$ charging. The cathode height was $6 \mu \mathrm{m}$.
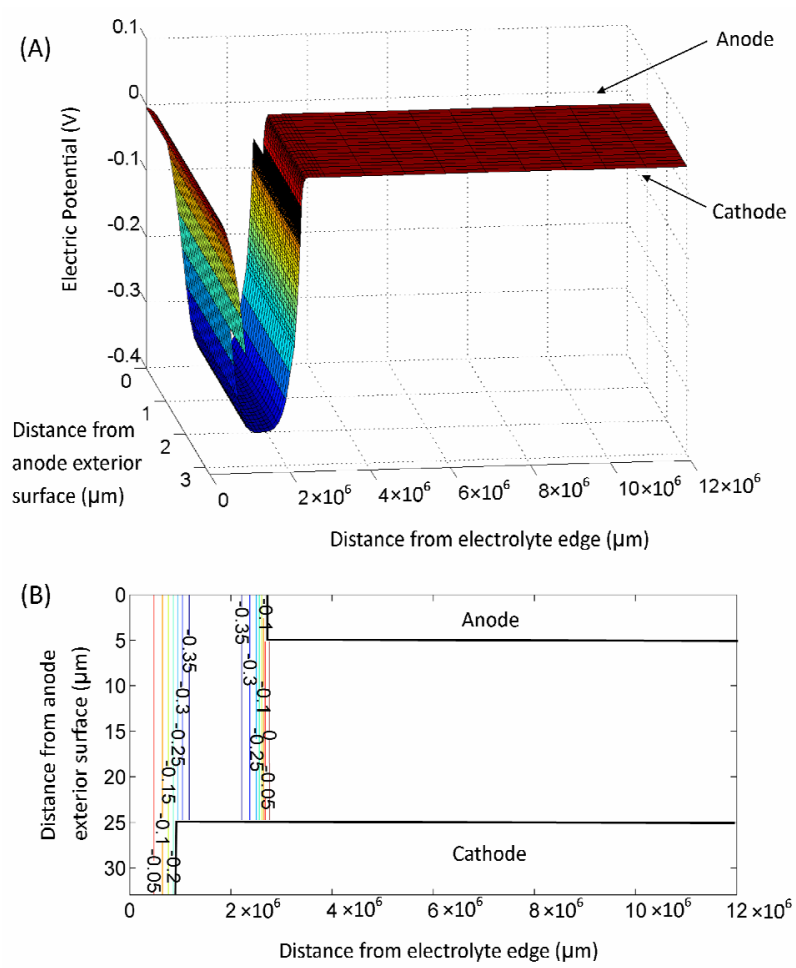

Figure 14. Predicted electric potential field (V) in the electrolyte for the case of a $1.75 \mathrm{~cm}$ cathode extension after 100 seconds of charging as (A) a surface plot and (B) a contour plot with cell geometry overlay. The cathode height was 8 $\mu \mathrm{m}$ and the cathode length extension was $1.75 \mathrm{~cm}$.

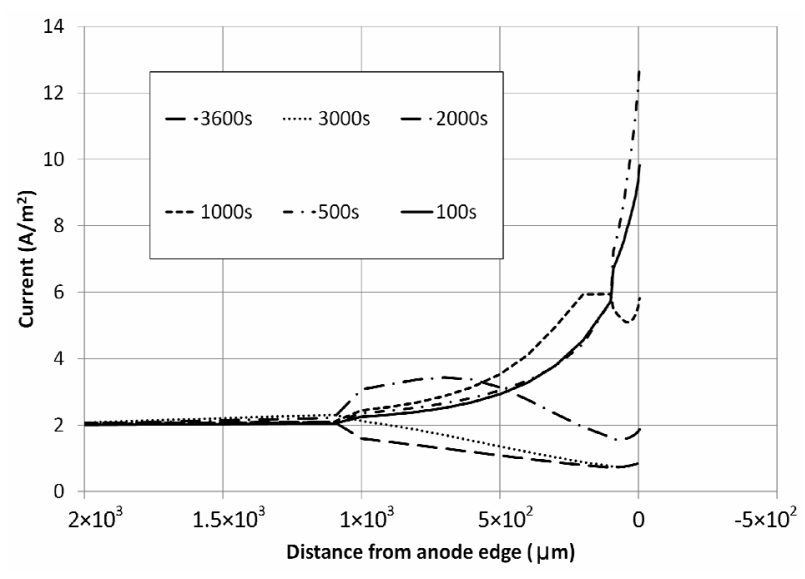

Figure 15. Predicted electric current emanating from anode surface at different times for the case of a $1.75 \mathrm{~cm}$ cathode extension. Positive distance values represent the interior anode surface and negative distance values represent the distance along the anode edge itself, away from the anode tip.

rent from this region for two reasons: this region was the closest to the extended cathode and this region had a greater surface area due to the anode edge. Also, from Figure 2 it is evident that significant concentration gradients were possible in the initially charging anode that 
did not cause significant gradients of equilibrium potential. Figure 16 shows a figure similar to Figure 15, but describing the current inserted into the cathode. At the early charging times of $100 \mathrm{~s}$ and $500 \mathrm{~s}$, Figure 16 shows that current densities of approximately 0.4 and $0.2 \mathrm{~A} / \mathrm{m}^{2}$ were inserted along the extended region of the cathode, respectively. At early charging times the gradient of lithium in the cathode caused significant gradients of equilibrium potential which provided the driving force behind the large potential drop in the electrolyte (shown in Figure 14), and caused these significant cathodic reaction rates towards the edge (shown in Figure 16). Over an initial period of time, small amounts of lithium were inserted into the extended cathode region and the concentration of lithium in the extended cathode region increased such that a large difference in equilibrium potential no longer existed between the edge region and the bulk cell region (see Figure 2). This decrease in the difference in equilibrium potential decreased the available driving force for the migration and insertion of lithium along the length of the extended cathode and is visible in Figure 16 that shows how the current drawn by the extended cathode decreased for times of $1000 \mathrm{~s}$ and later. This decrease in current drawn by the extended cathode also affected the current emanating from the anode. Figure 15 shows that after $1000 \mathrm{~s}$ the excess current drawn from the region towards the edge of the anode had decreased and some other effects were visible as the "hook" shape in the reaction rates towards the edge region. This hook shape was likely because of the decreased current being drawn towards the extended cathode and the decreased Ohmic drop in the electrolyte making it more possible for current to be drawn from the anode surface further into the cell. This current drawn from the anode further into the cell took advantage of the fact that the anode edge became more depleted of lithium during the early charging when the extended cathode was drawing significant quantities of current. Figure 15 shows that as time progressed further, less and less excess current was produced towards the anode edge region, and instead, because the extended cathode was no longer drawing significant current, the concentration gradients previously established in the anode became the dominant phenomenon impacting the rates of anodic reactions. This was because, as the cell became more charged and the stoichiometric coefficient in the anode decreased, a slight electrochemical potential gradient is evident towards the left of the corresponding solid line in Figure 2. This significant lithium concentration gradient in the anode after 1 hour is shown in Figure 17. Figure 18 shows the lithium concentration gradient in the cathode after 1 hour. It can be seen that the concentration of lithium in the extended region was approximately one tenth of the maximum value seen in the bulk cell.

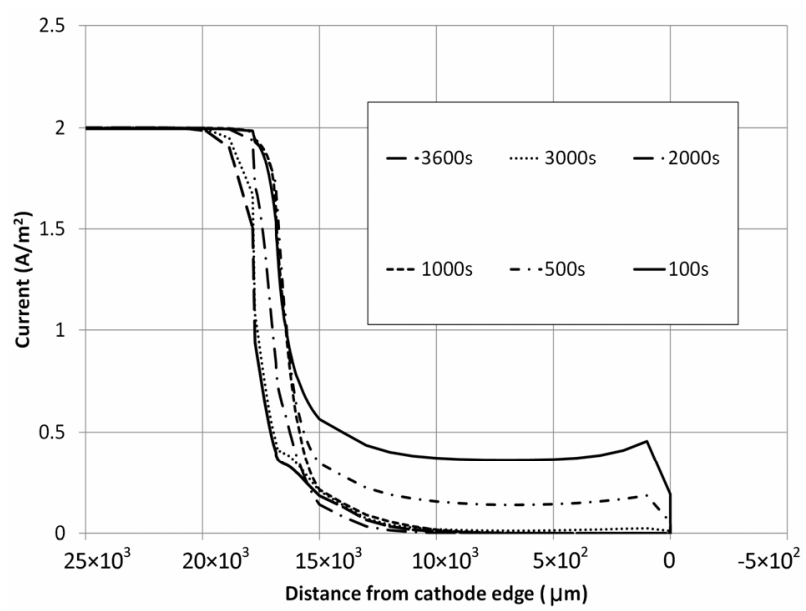

Figure 16. Predicted electric current inserted into cathode surface at different times for the case of a $1.75 \mathrm{~cm}$ cathode extension. Positive distance values represent the interior cathode surface and negative distance values represent the distance along the cathode edge itself, away from the cathode tip.

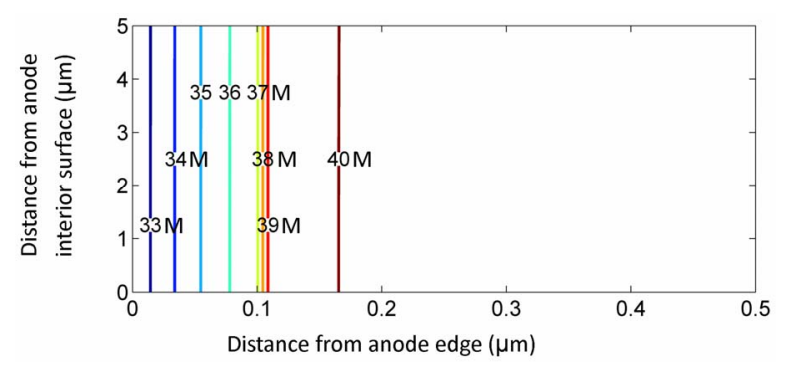

Figure 17. Predicted anode concentration for the case of a $1.75 \mathrm{~cm}$ cathode extension after 1 hour of charging $2 \mathrm{~A} / \mathrm{m}^{2}$.

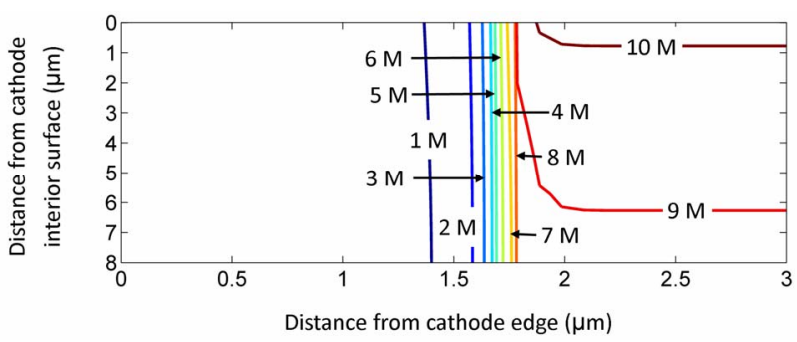

Figure 18. Predicted cathode concentration for the case of a $1.75 \mathrm{~cm}$ cathode extension after 1 hour of charging at 2 $\mathbf{A} / \mathbf{m}^{2}$.

\section{Conclusions}

This paper explores the edge effects of electrodes in lithium-ion cells undergoing charging, and the effects of stoichiometric coefficient gradients within electrodes. It was predicted, for the cases examined, that the increase in effective conductivity associated with a flooded electrolyte that is extended past the electrode edges does not have an appreciable effect on the rates of anodic or cathodic reactions near the edge regions. However, it was 
predicted that lithium concentration gradients inside the cathode impact the rate of cathodic reactions significantly and concentration gradients inside the anode do not significantly impact the rate of anodic reactions, both during early cell charging. Instead, the rates of anodic reactions are significantly affected by the surface area of the anode contacting the electrolyte, and not the concentration gradient of lithium in the anode. It was also predicted that during later stages of cell charging, when the gradient of equilibrium potential due to a gradient in cathodic stoichiometric coefficient is less steep, concentration gradients within the cathode (for equal electrode lengths) are more likely and might lead to a possibility for lithium deposition at the cathode edge region.

Simulations were conducted for the case where the cathode edge was extended past the anode edge to reduce the possibility for lithium deposition at the cathode edge region. The simulations indicate that the stoichiometric coefficient of lithium in an extended cathode edge would be reduced in value; however, this extension may cause a large electric potential drop along the electrolyte length (during early cell charging) that corresponds to the lithium stoichiometric coefficient gradient in the extended cathode and also with the Ohmic losses and concentration gradients within the electrolyte itself. It was observed that this equilibrium potential gradient would decrease as charging of the cell proceeded, causing a reduction in the rate of cathodic reactions occurring along the extended cathode region. This reduction in the rate of cathodic reactions along the extended cathode region reduces the risk for lithium deposition at the cathode edge region, as desired by many cell manufacturers.

\section{Acknowledgements}

The authors thank the University of Saskatchewan for computing facilities and the National Science and Engineering Research Council for a Canada Doctoral Scholarship.

\section{REFERENCES}

[1] E. Scott, G. Tam, B. Anderson and C. Schmidt, "Anomalous Potentials in Lithium Ion Cells: Making the Case for 3-D Modeling of 3-D Systems," The Electrochemical Society Meeting, Orlando, 13 October 2003.

[2] E. Scott, G. Tam, B. Anderson and C. Schmidt, "Observation and Mechanism of Anomalous Local Potentials during Charging of Lithium Ion Cells," The Electrochemical Society Meeting, Paris, 29 April 2003.
[3] K. West, T. Jacobsen and S. Atlung, "Modeling of Porous Insertion Electrodes with Liquid Electrolyte," Journal of the Electrochemical Society, Vol. 129, No. 7, 1982, pp. 1480-1485. doi:10.1149/1.2124188

[4] M. Doyle, T. F. Fuller and J. Newman, "Modeling of Galvanostatic Charge and Discharge of the Lithium/Polymer/Insertion Cell," Journal of the Electrochemical Society, Vol. 140, No. 6, 1993, pp. 1526-1533. doi:10.1149/1.2221597

[5] T. F. Fuller, M. Doyle and J. Newman, "Simulation and Optimization of the Dual Lithium Ion Insertion Cell," Journal of the Electrochemical Society, Vol. 141, No. 1, 1994, pp. 1-10. doi:10.1149/1.2054684

[6] P. Arora, M. Doyle and R. E. White, "Mathematical Modeling of the Lithium Deposition Overcharge Reaction in Lithium-Ion Batteries Using Carbon-Based Negative Electrodes," Journal of the Electrochemical Society, Vol. 146, No. 10, 1999, pp. 3543-3553. doi:10.1149/1.1392512

[7] M. Tang, P. Albertus and J. Newman, "Two-Dimensional Modelling of Lithium Deposition during Cell Charging," Journal of the Electrochemical Society, Vol. 156, No. 5, 2009, pp. A390-A399. doi:10.1149/1.3095513

[8] K. Eberman, P. M. Gomadam, G. Jain and E. Scott, "Material and Design Options for Avoiding Lithium-Plating during Charging," ECS Transactions, Vol. 25, No. 35, 2010, pp. 47-58. doi:10.1149/1.3414003

[9] G. F. Kennell and R. W. Evitts, "Charge Density in NonIsotropic Electrolytes Conducting Current," The Canadian Journal of Chemical Engineering, Vol. 90, No. 2, 2012, pp. 377-384.

[10] W. Dreyer, M. Gaberscek, C. Guhlke, R. Huth and J. Jamnik, "Phase Transition in a Rechargeable Lithium Battery," European Journal of Applied Mathematics, Vol. 22, No. 3, 2011, pp. 267-290. doi:10.1017/S0956792511000052

[11] G. F. Kennell, "Electrolytic Transport, Electric Fields, and the Propensity for Charge Density in Electrolytes," Ph.D. Dissertation, University of Saskatchewan, Saskatoon, 2011.

[12] M. Doyle and Y. Fuentes, "Computer Simulations of a Lithium-Ion Polymer Battery and Implications for Higher Capacity Next-Generation Battery Designs," Journal of the Electrochemical Society, Vol. 150, No. 6, 2003, pp. A706-A713. doi:10.1149/1.1569478

[13] J. Christensen, V. Srinivasan and J. Newman, "Optimization of Lithium-Titanate Electrodes for High-Power Cells," Journal of the Electrochemical Society, Vol. 153, No. 3, 2006, pp. A560-A565. doi:10.1149/1.2172535

[14] S. G. Stewart and J. Newman, "The Use of UV/Vis Absorption to Measure Diffusion Coefficients in LiPF6 Electrolytic Solutions," Journal of the Electrochemical Society, Vol. 155, No. 1, 2008, pp. F13-F16. doi:10.1149/1.2801378 


\section{Nomenclature}

$C$ species concentration $\left(\mathrm{mol} / \mathrm{m}^{3}\right)$

$C_{s}$ solid phase lithium concentration $\left(\mathrm{mol} / \mathrm{m}^{3}\right)$

$C_{t}$ maximum lithium concentration $\left(\mathrm{mol} / \mathrm{m}^{3}\right)$

$D$ effective diffusion coefficient $\left(\mathrm{m}^{2} / \mathrm{s}\right)$

$D_{0}$ diffusion coefficient of liquid phase $\left(\mathrm{m}^{2} / \mathrm{s}\right)$

$F$ Faraday's Constant $(96,487 \mathrm{C} / \mathrm{mol})$

$i$ current density $\left(\mathrm{A} / \mathrm{m}^{2}\right)$

$i$ current density vector $\left(\mathrm{A} / \mathrm{m}^{2}\right)$

$i_{L i}$ current density of insertion/dissolution $\left(\mathrm{A} / \mathrm{m}^{2}\right)$

$k$ lithium insertion rate constant $\left(\mathrm{m}^{2.5} \mathrm{~mol}^{-0.5} \mathrm{~s}^{-1}\right)$

$I_{\text {set }}$ applied current density $\left(\mathrm{A} / \mathrm{m}^{2}\right)$

$l$ length $(\mathrm{m})$

$l_{\text {electrode }}$ length of electrode $(\mathrm{m})$

$R$ gas constant $\left(8.314 \mathrm{JK}^{-1} \mathrm{~mol}^{-1}\right)$

$S$ source term $\left(\mathrm{mol} / \mathrm{m}^{3} \cdot \mathrm{s}\right)$

$s$ position (m)

$t$ time (s)

$T$ temperature (K) $u$ mobility $\left(\mathrm{m}^{2} \cdot \mathrm{mol} / \mathrm{J} \cdot \mathrm{s}\right)$

$U$ equilibrium potential $(\mathrm{V})$

$\mathrm{x}$ position along the $\mathrm{x}$-dimension $(\mathrm{m})$

$z$ charge number

\section{Greek Letters}
$\alpha$ transfer coefficient
$\varepsilon$ volume fraction
$\Phi$ electric potential (V)
$\kappa$ conductivity $(\mathrm{C} / \mathrm{Vms})$

\section{Subscripts/Superscripts}
$a$ anode
$c$ cathode
$e$ electrolyte
$i$ species 